\title{
Investigation of mean platelet volume as a predictor of acute versus chronic immune thrombocytopenic purpura in children: An exploratory analysis
}

\author{
Jessica Keim-Malpass ${ }^{* 1,2}$, Jenifer L. Lavigne ${ }^{2}$, Jennifer Pope $^{2}$, Javier Oesterheld ${ }^{2}$ \\ ${ }^{1}$ University of Virginia School of Nursing, Charlottesville, VA, USA \\ ${ }^{2}$ Levine Children's Hospital, Division of Pediatric Hematology/Oncology, Charlotte, NC, USA
}

Received: December 1, 2014

Accepted: March 8, 2015

Online Published: March 18, 2015

DOI: $10.5430 /$ cns.v3n3p23

URL: http://dx.doi.org/10.5430/cns.v3n3p23

\begin{abstract}
Current difficulty remains in predicting acute versus chronic immune thrombocytopenic purpura (ITP) in children at the time of diagnosis. The purpose of this exploratory retrospective study was to determine the relationship between mean platelet value (MPV) and initial platelet count at time of diagnosis on prediction of acute versus chronic ITP among pediatric patients. When odds ratios were calculated, initial platelet count less than $10,000 / \mu \mathrm{l}$ was the only statistically significant predictor of acute ITP (i.e., case resolution). MPV $<8 \mathrm{fl}$ was not a statistically significant predictor of acute ITP, although clinical trends suggested that those with acute ITP had lower MPV at time of diagnosis. This exploratory study does suggest that multi-center prospective study designs are needed for further study to describe if there is a relationship between MPV in determining acute versus chronic ITP. Adequately predicting acute versus chronic ITP at diagnosis could have numerous implications for children and families as they prepare for treatment and help nurses deliver patient-centered interventions.
\end{abstract}

Key Words: Platelet, Immune thrombocytopenic purpura, Pediatric, Hematology

\section{INTRODUCTION}

Idiopathic (or immune) thrombocytopenic purpura (ITP) is a hemorrhagic disorder described by low platelet counts, spontaneous bruising, purpuric or petechial rash, and mucosal bleeding and is often defined as one platelet count $\leq 100,000 / \mu 1 .^{[1,2]}$ ITP affects approximately 100 people per 1 million per year in the general population and about half of these cases occur in children. ${ }^{[3,4]}$ ITP is caused by antibody-mediated platelet destruction and impaired platelet production resulting in thrombocytopenia ${ }^{[5]}$ and can be an acute illness or chronic disorder depending on the duration of thrombocytopenia. ${ }^{[6]}$ Generally, ITP is classified as "acute" if thrombocytopenia lasts less than 6 months after diagno- sis and "chronic" if thrombocytopenia lasts greater than 6 months after initial presentation. ${ }^{[3,6,7]}$ Recent consensus statements have further refined the definition of ITP to include "newly diagnosed ITP" from the time of diagnosis to 3 months, "persistent" if the ITP lasts 3-12 months from diagnosis and "chronic" if the disease remains in duration greater than 12 months and occurrence of spontaneous remission becomes less likely. ${ }^{[8]}$ Even so, these definitions are not consistently used among registered ITP clinical trials or in clinical practice. ${ }^{[8]}$ Additionally, the revised definitions may be less clinically meaningful for parents and families whose primary interest is in the likelihood of remission.

ITP in children is more likely to be acute ${ }^{[6]}$ and is usually USA.

\footnotetext{
*Correspondence: Jessica Keim-Malpass; Email: Jlk2t@ virginia.edu; Address: University of Virginia School of Nursing, Charlottesville, VA, 22908,
} 
benign and self-limiting. ${ }^{[7]}$ However, one-third of childhood ITP becomes a long-term disease due to both chronic and relapsing forms. ${ }^{[9]}$ There are several treatment options for ITP including corticosteroids, intravenous immunoglobulin (IVIG), Anti-D immunoglobulin (Win-Rho), and TPO receptor agonists, however predicting clinical outcome based on drug treatment remains unclear. ${ }^{[5,10]}$

Unlike adults, the majority of children with ITP have a favorable prognosis of spontaneous remission. In roughly 80 percent of cases, ITP resolves within 6 months irrespective of treatment decisions. ${ }^{[11]}$ Therefore, difficulty exists in predicting prognosis at diagnosis for children. ${ }^{[3]}$ Previous research has demonstrated there are several factors that predict chronic disease: insidious presentation (i.e., symptoms for more than 2 weeks), female sex, no mucosal bleeding on presentation, lack of viral disease preceding presentation, and initial diagnosis greater than 10 years of age. ${ }^{[1,12,13]}$

Mean platelet volume (MPV) has been associated as a prognostic indicator for chronicity of disease in other adult hematologic disease processes. ${ }^{[14,15]}$ To the authors' knowledge, only one previous investigator has assessed the utility of MPV as a predictive factor for chronic ITP in children. ${ }^{[3]}$ Ahmed and colleagues demonstrated through univariate analysis that low admission MPV ( $<8 \mathrm{fl})$, history of viral prodrome, and low admission platelet count $(<10,000 / \mu \mathrm{l})$ all independently predicted a favorable outcome of acute ITP. ${ }^{[3]}$ While other studies have not specifically assessed admission MPV, they have found associations between low admission platelet count $(<10,000 / \mu \mathrm{l})$ and resolution of disease, thus acute ITP. ${ }^{[1,3,4,9,13,16-19]}$

Clarification of the utility of MPV as a potential prognostic marker is important in pediatric ITP for prediction of a acute or chronic condition, especially considering its convenience and low cost of this clinical laboratory assessment. Nurses are often faced with educating families of children with ITP during the time of initial diagnosis, and being able to predict whether the ITP diagnosis is chronic or self-limiting has many anticipated benefits. The purpose of this exploratory study was to determine the relationship between MPV and initial platelet count on index admission and prediction of acute versus chronic ITP among pediatric patients at a single Children's Hospital in the United States. Our hypothesis was clinical presentation with MPV values less than $8 \mathrm{fl}$ at the time of initial ITP diagnosis could predict acute ITP or resolution of the disease in our pediatric ITP population.

\section{Methods}

The study protocol was approved by Carolinas Medical Center Institutional Review Board. Medical records of all pediatric patients who met eligibility were included. Inclusion criteria included: children age 18 or below who were diagnosed and treated with ITP in the study time frame as an initial new diagnosis, with available blood counts at the time of diagnosis and subsequent follow-up visits. Those patients who did not have meet eligibility included: thrombocytopenia secondary to chemotherapy or other underlying hematologic condition, or no available documentation of resolution of the case (acute vs. chronic ITP) either through clinic follow-up visit or follow-up platelet counts and were excluded from the analysis. We retrospectively reviewed medical record data on 63 patients with ITP diagnosed from January 2007 to June 2013 at Levine Children's Hospital in Charlotte, NC. Twenty-one patients were excluded because either there was no initial MPV at diagnosis, and/or they were missing follow-up counts during the first 12 months post-diagnosis. Our final sample with complete data yielded 42 patients.

\subsection{Case ascertainment}

Verification of ITP was made through ICD-9 code and chart review of the index admission or outpatient visit. Index platelet count (i.e., platelet count at time of ITP diagnosis), MPV, and treatments administered were all abstracted from medical records at the time of diagnosis. Index platelet count was dichotomized into the following categories: $<10,000 / \mu 1$ or $10,000-50,000 / \mu 1$. MPV was assessed both as a continuous variable and dichotomized into $<8 \mathrm{fl}$ or $\geq 8 \mathrm{fl}$. ITP status (acute versus chronic) was determined at the 6-month follow-up visit as documented by the physician's note and lab values. We used the classic definition of acute ITP lasting less than 6 months in duration and chronic ITP lasting greater than 6 months in duration. Demographic variables such as gender, age, race/ethnicity as well as initial treatment variables (corticosteroid and IVIG use) were also ascertained from the medical record.

\subsection{Analysis}

Descriptive statistics were calculated to describe all variables using mean/standard deviation for continuous variables or percent/frequency for categorical variables. Variables associated with acute versus chronic ITP status were then compared using the chi-square statistics (and Fisher's exact test when $n<10$ ) for categorical variables and t test for continuous variables with normal distributions. Independent associations between (1) MPV, (2) index platelet count, (3) treatment using corticosteroids, and (4) treatment using IVIG on the clinical outcome of acute or chronic ITP status were then calculated. Odds ratios (OR), confidence intervals (CI) and $p$ values were reported to determine the impact of MPV $(<8 \mathrm{fl} v s . \geq 8 \mathrm{fl})$, index platelet count $(<10,000 / \mu \mathrm{l}$ vs. $\geq 10,000 / \mu \mathrm{l}$ ), treatment with corticosteroids (yes/no) and 
treatment with IVIG (yes/no) on risk associated with ITP SPSS version 21.0, IBM.

status (acute versus chronic). Statistics were calculated with

Table 1. Patient characteristics for acute vs. chronic ITP (means/SD and frequency/percentages)

\begin{tabular}{|c|c|c|c|c|}
\hline Variable & Total $\mathrm{N}=42$ & Acute ITP N = 29 & Chronic ITP N = 13 & $P$ value \\
\hline Age (years) & 4.83 (3.99), range $<1$ yr-15 yrs & $4.83(4.22)$ & $4.85(3.58)$ & .61 \\
\hline Sex & & & & .06 \\
\hline Male & $15(35.7 \%)$ & $10(34.5 \%)$ & $5(38.5 \%)$ & \\
\hline Female & $27(64.3 \%)$ & $19(65.5 \%)$ & $8(61.5 \%)$ & \\
\hline Race & & & & .56 \\
\hline White & $24(57.1 \%)$ & $18(62.1 \%)$ & $6(46.2 \%)$ & \\
\hline Other & $9(21.4 \%)$ & $5(17.2 \%)$ & $4(30.8 \%)$ & \\
\hline Ethnicity & & & & .28 \\
\hline Hispanic & $6(14.3 \%)$ & $4(13.8 \%)$ & $2(15.4 \%)$ & \\
\hline Non-Hispanic & $31(73.8)$ & $20(69.0 \%)$ & $11(84.6 \%)$ & \\
\hline Unknown & $5(11.9 \%)$ & $5(17.2 \%)$ & $0(0 \%)$ & \\
\hline MPV at diagnosis & $8.95(1.71)$, range $5.4-12.1$ & $8.88(1.49)$ & $9.09(2.18)$ & .22 \\
\hline Platelet count at diagnosis & & & & $.04^{*}$ \\
\hline $10,000-50,000 / \mu \mathrm{l}$ & $16(38.1 \%)$ & $8(27.6 \%)$ & $8(61.5 \%)$ & \\
\hline Initial treatment & & & & .64 \\
\hline Corticosteroids & $3(7.1 \%)$ & $2(6.9 \%)$ & $1(7.7 \%)$ & \\
\hline IVIG & $36(85.7 \%)$ & $25(86.2 \%)$ & $11(84.6 \%)$ & \\
\hline None & $3(7.1 \%)$ & $2(6.9 \%)$ & $1(7.7 \%)$ & \\
\hline
\end{tabular}

*Indicates a statistically significant finding at $p<.05$

\section{RESUlts}

A total of 42 patients met inclusion criteria for this study. These patients had a mean age of nearly 5 years (range $<1$ year to 15 years) and were majority female $(64 \%)$ and white (57\%). The mean MPV at diagnosis was 8.95 (range 5.412.1) and mean MPV was higher in the chronic ITP group (9.09) compared to the acute ITP group (8.88). The majority of the study population had an initial platelet count of less than $10,000 / \mu 1(62 \%)$ (see Table 1$)$. The only statistically significant difference between acute versus chronic ITP was in platelet count at diagnosis $(p=.04)$ (see Table 1). A higher percentage of those with acute ITP presented with an initial platelet count less than $10,000 / \mu \mathrm{l}(72 \%)$ and a higher percentage of patients with chronic ITP presented with platelet count between 10,000 and $50,000 / \mu \mathrm{l}(62 \%)$.

Table 2. Odds ratio of acute ITP (i.e. case resolution)

\begin{tabular}{lll}
\hline Variable & OR/CI & P value \\
\hline MPV $<8$ fl & $1.14(0.21-3.59)$ & .83 \\
Initial platelet $<10,000 / \mu \mathrm{l}$ & $4.20(1.05-16.74)^{*}$ & $.04^{*}$ \\
Corticosteroid treatment & $1.13(0.09-13.64)$ & .92 \\
IVIG treatment & $0.88(0.14-5.54)$ & .89 \\
\hline
\end{tabular}

Indicates a statistically significant finding

When odds ratios were calculated, initial platelet count less than $10,000 / \mu \mathrm{l}$ was the only statistically significant predictor of acute ITP (i.e., case resolution). Presenting with an initial platelet count less than $10,000 / \mu \mathrm{l}$ at diagnosis led to 4.2 times the likelihood of acute ITP versus chronic ITP (OR 4.2, CI 1.05-16.74, $p$ value $=.04)($ see Table 2$)$. MPV $<8$ fl (OR 1.14, CI 0.21-3.59), corticosteroid treatment (OR 1.13, CI 0.09-13.64), and IVIG treatment (OR 0.88, CI 0.14-5.54) did not yield statistically significant findings in prediction of acute ITP (see Table 2). Our hypothesis that MPV < $8 \mathrm{fl}$ could predict acute ITP was not substantiated in this exploratory analysis.

\section{Conclusions}

This study adds to the evidence that pediatric presentation of platelet count less than $10,000 / \mu 1$ at the time of diagnosis can be used as a predictor of acute ITP and subsequent case resolution. ${ }^{[1,3,4,9,13,16-19]}$ Although not statistically significant, our study found that presentation of MPV less than $8 \mathrm{fl}$ demonstrated a slightly higher odds of acute ITP diagnosis. Our analysis likely did not yield a large enough sample size to adequately answer the MPV prediction or any treatment related factors with statistical significance. Additionally, findings are based on a single Children's Hospital may lack generalizability. Due to the changing ITP definition, further study is also warranted to determine if there are group differences in MPV and platelet count at presentation among the new diagnostic categories.

This exploratory study does suggest that multi-center 
prospective study designs are needed to adequately determine that MPV can be used as an independent predictor of acute vs. chronic ITP. Nordic researchers Edslev and colleagues ${ }^{[17]}$ developed and validated a clinical score predicting acute ITP and case resolution based on six clinical features: abrupt onset, age less than 10 years, proceeding infection, platelet count less than $5000 / \mu 1$, wet purpura and male gender. With further study, it is possible that MPV could provide additional clinical utility of ITP prediction in a cost effective manner and be included in clinical prediction strategies and nursing education strategies.

Adequately predicting acute versus chronic ITP at diagnosis would have numerous implications for children and families as they prepare for treatment. Nurses must provide a wide range of education and coordination when caring for children with ITP, including; education about treatment, side effects, the frequency of laboratory assessment, and on the need for ongoing self-assessment of bleeding, bruising and petechiae. ${ }^{[20]}$ Because of the dramatic prognostic uncertainty at the time of diagnosis, obtaining more accurate prognostic strategies can dramatically improve the tailored care, coordination and education nurses can provide.

\section{CONFLicts OF InTEREST Disclosure}

The authors declare that there is no conflict of interests regarding the publication of this paper.

\section{REFERENCES}

[1] Glanz J, France E, Xu S, et al. A population-based, multisite cohort study of the predictors of chronic idiopathic thrombocytopenic purpura in children. Pediatrics. 2008 Mar; 121(3): e506-12.

[2] Neunert CE. Current management of immune thrombocytopenia Hematology. 2013 Jan; 2013: 276-82.

[3] Ahmed S, Siddiqui AK, Shahid RK, et al. Prognostic variables in newly diagnosed childhood immune thrombocytopenia. Am J Hematol. 2004 Dec; 77(4): 358-62.

[4] Zeller B, Rajantie J, Hedlund-Treutiger I, et al. Childhood idiopathic thrombocytopenic purpura in the Nordic countries: Epidemiology and predictors of chronic disease. Acta Paediatr. 2005 Feb 1; 94(2) 178-84.

[5] Khan M, Mikhael J. A review of immune thrombocytopenic purpura focus on the novel thrombopoietin agonists. J Blood Med. 2010 Jan; 1: 21-31.

[6] Nugent DJ. Immune thrombocytopenic purpura of childhood. Hematology Am Soc Hematol Educ Program. 2006 Jan; 97-103. PMID:17124046. http://dx.doi.org/10.1182/asheducatio $\mathrm{n}-2006.1 .97$

[7] Shad AT, Gonzalez CE, Sandler SG. Treatment of immune thrombocytopenic purpura in children: current concepts. Paediatr Drugs. 2005 Jan; 7(5): 325-36. PMID:16220997. http://dx.doi .org/1 0.2165/00148581-200507050-00004

[8] Michel M. Immune Thrombocytopenia Nomenclature, Consensus Reports, and Guidelines: What Are the Consequences for Daily Practice and Clinical Research? Semin Hematol. 2013; 50(1): S50-4. PMID:23664517. http://dx.doi.org/10.1053/j.seminhema tol.2013.03.008

[9] Tamminga R, Berchtold W, Bruin M, et al. Possible lower rate of chronic ITP after IVIG for acute childhood ITP an analysis from registry I of the Intercontinental Cooperative ITP Study Group (ICIS). Br J Haematol. 2009 Jul; 146(2): 180-4

[10] Imbach P, Kühne T, Müller D, et al. Childhood ITP: 12 months follow-up data from the prospective registry I of the Intercontinental Childhood ITP Study Group (ICIS). Pediatr Blood Cancer. 2006 Mar; 46(3): 351-6.
[11] Nugent DJ. Childhood immune thrombocytopenic purpura. Blood Rev. 2002 Mar; 16(1): 27-9.

[12] Robb LG, Tiedeman K. Idiopathic thrombocytopenic of chronic disease. Arch Dis Child. 1990; 65: 502-6. http://dx.doi.org/10. 1136/adc.65.5.502

[13] Donato H, Picon A, Martinez M, et al. Demographic Data, Natural History, and Prognostic Factors of Idiopathic Thrombocytopenic Purpura in Children: A Multicentered Study From Argentina. Pediatr Blood Cancer. 2009; 52: 491-6. PMID:19058214. http://dx.doi.org/10.1002/pbc. 21872

[14] Baynes RD, Lamparelli RD V, Chetty N, et al. Platelet parameters: Part II. Platelet volume-number relationships in various normal and disease states. South African Med J. 1988; 73(9): 39-43. PMID:3340900.

[15] Threatte GA. Usefulness of the mean platelet volume. Clin Lab Med. Department of Pathology, State University of New York, Health Science Center, Syracuse; 1993 Dec; 13(4): 937-50.

[16] Bruin M, Bierings M, Uiterwaal C, et al. Platelet count, previous infection and FCGR2B genotype predict development of chronic disease in newly diagnosed idiopathic thrombocytopenia in childhood: results of a prospective study. Br J Haematol. 2004 Dec; 127(5): 561-7.

[17] Edslev PW, Rosthøj S, Treutiger I, et al. A clinical score predicting a brief and uneventful course of newly diagnosed idiopathic thrombocytopenic purpura in children. Br J Haematol. 2007 Aug; 138(4): 513-6.

[18] Jayabose S, Levendoglu-Tugal O, Ozkaynkak M, et al. LongTerm Outcome of Chronic Idiopathic Thrombocytopenic Purpura in Children. J Pediatr Hematol Oncol. 2004; 26(11): 724-6. PMID:15543006. http://dx.doi.org/10.1097/00043 426-200411000-00007

[19] Watts RG. Idiopathic Thrombocytopenic Purpura: A 10-Year Natural History Study at the Childrens Hospital of Alabama. Clin Pediatr (Phila). 2004 Oct 1; 43(8): 691-702.

[20] Winkeljohn D. Diagnosis, Treatment, and Management of Immune Thrombocytopenia. Clin J Oncol Nurs. 2013; 17(6): 664-6. PMID:24305488. http://dx.doi.org/10.1188/13.CJON. 664 $-666$ 